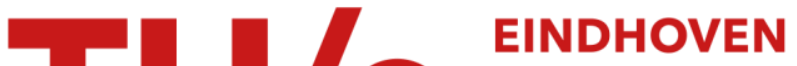 UNIVERSITY OF TECHNOLOGY
}

\section{A hybrid stochastic-deconvolution model for LES of particle- laden flow}

Citation for published version (APA):

Michalek, W. R., Kuerten, J. G. M., Zeegers, J. C. H., Liew, R., Pozorski, J., \& Geurts, B. J. (2013). A hybrid stochastic-deconvolution model for LES of particle-laden flow. Physics of Fluids, 25(12), 123302-1/15. https://doi.org/10.1063/1.4849536

DOI:

$10.1063 / 1.4849536$

Document status and date:

Published: 01/01/2013

\section{Document Version:}

Publisher's PDF, also known as Version of Record (includes final page, issue and volume numbers)

\section{Please check the document version of this publication:}

- A submitted manuscript is the version of the article upon submission and before peer-review. There can be important differences between the submitted version and the official published version of record. People interested in the research are advised to contact the author for the final version of the publication, or visit the $\mathrm{DOI}$ to the publisher's website.

- The final author version and the galley proof are versions of the publication after peer review.

- The final published version features the final layout of the paper including the volume, issue and page numbers.

Link to publication

\section{General rights}

Copyright and moral rights for the publications made accessible in the public portal are retained by the authors and/or other copyright owners and it is a condition of accessing publications that users recognise and abide by the legal requirements associated with these rights.

- Users may download and print one copy of any publication from the public portal for the purpose of private study or research.

- You may not further distribute the material or use it for any profit-making activity or commercial gain

- You may freely distribute the URL identifying the publication in the public portal.

If the publication is distributed under the terms of Article 25fa of the Dutch Copyright Act, indicated by the "Taverne" license above, please follow below link for the End User Agreement:

www.tue.nl/taverne

Take down policy

If you believe that this document breaches copyright please contact us at:

openaccess@tue.nl

providing details and we will investigate your claim. 


\section{AIP | Physics of Fluids}

A hybrid stochastic-deconvolution model for large-eddy simulation of particle-laden flow

W. R. Michaek, J. G. M. Kuerten, J. C. H. Zeegers, R. Liew, J. Pozorski, and B. J. Geurts

Citation: Physics of Fluids (1994-present) 25, 123302 (2013); doi: 10.1063/1.4849536

View online: http://dx.doi.org/10.1063/1.4849536

View Table of Contents: http://scitation.aip.org/content/aip/journal/pof2/25/12?ver=pdfcov

Published by the AIP Publishing

\section{AlP Re-register for Table of Content Alerts}

Create a profile. 困 Sign up today! 


\title{
A hybrid stochastic-deconvolution model for large-eddy simulation of particle-laden flow
}

\author{
W. R. Michałek, ${ }^{1, \text { a) }}$ J. G. M. Kuerten, ${ }^{1,2}$ J. C. H. Zeegers, ${ }^{3}$ R. Liew, ${ }^{3}$ \\ J. Pozorski, ${ }^{4}$ and B. J. Geurts ${ }^{2,3}$ \\ ${ }^{1}$ Department of Mechanical Engineering, Eindhoven University of Technology, \\ 5600 MB Eindhoven, The Netherlands \\ ${ }^{2}$ Faculty EEMCS, University of Twente, 7500 AE Enschede, The Netherlands \\ ${ }^{3}$ Department of Applied Physics, Eindhoven University of Technology, \\ 5600 MB Eindhoven, The Netherlands \\ ${ }^{4}$ Institute of Fluid-Flow Machinery, Polish Academy of Sciences, Gdansk, Poland
}

(Received 24 May 2013; accepted 3 December 2013; published online 20 December 2013)

\begin{abstract}
We develop a hybrid model for large-eddy simulation of particle-laden turbulent flow, which is a combination of the approximate deconvolution model for the resolved scales and a stochastic model for the sub-grid scales. The stochastic model incorporates a priori results of direct numerical simulation of turbulent channel flow, which showed that the parameters in the stochastic model are quite independent of Reynolds and Stokes number. In order to correctly predict the flux of particles towards the walls an extra term should be included in the stochastic model, which corresponds to the term related to the well-mixed condition in Langevin models for particle dispersion in inhomogeneous turbulent flow. The model predictions are compared with results of direct numerical simulation of channel flow at a frictional Reynolds number of 950 . The inclusion of the stochastic forcing is shown to yield a significant improvement over the approximate deconvolution model for the particles alone when combined with a Stokes dependent weight-factor for the well-mixed term. (c) 2013 AIP Publishing LLC. [http://dx.doi.org/10.1063/1.4849536]
\end{abstract}

\section{INTRODUCTION}

Turbulent particle-laden flows occur frequently in the environment and in industry. These flows have richer physics than flow of a single-phase fluid. In recent years numerical simulation methods have been developed for the study of such particle-laden turbulent flows. The most detailed simulation method requires the resolution of the flow down to the scales of the particles. This is only possible nowadays, if the number of particles is limited to several hundreds. ${ }^{1}$ For larger numbers of small particles the details of the flow around each particle cannot be resolved, and a point-particle approach is adopted, in which correlations for the forces exerted by the fluid on a particle are used. ${ }^{2}$ We will use the term direct numerical simulation (DNS), if in such a point-particle approach all scales of the fluid flow are resolved and no model for the smallest scales of the turbulence is used. However, DNS can only be performed at relatively low Reynolds numbers. ${ }^{3}$ In contrast, large-eddy simulation (LES) can be performed at higher Reynolds numbers. This is possible because in LES only the larger eddies are resolved, since a spatially filtered fluid velocity is adopted. ${ }^{3}$ The absence of the small scales in the resolved part of the flow requires a sub-grid scale model for the fluid.

The missing scales in LES not only influence the resolved scales of the fluid flow, but also affect the motion of small particles present in the flow. The effect of the small scales on the particle motion can only be neglected when the particle relaxation time is large compared to the time scales of the smallest resolved scales in LES. ${ }^{4}$ In such a case the filtered fluid velocity can be used in

\footnotetext{
a)Electronic mail: w.michalek@tue.nl
} 
the equation of motion of the particles instead of the real unfiltered fluid velocity. For smaller particles, however, the disregard of the smallest scales of fluid velocity in the particle motion leads to significant discrepancies in the prediction of turbulent dispersion and of turbophoresis, the mean motion of particles toward the walls in wall-bounded turbulent flows. ${ }^{5}$ In particular, when the particle relaxation time is of the same order of magnitude as the Kolmogorov time, the prediction of particle behavior deteriorates without modeling the effect of the sub-grid scales on the particles. ${ }^{5}$

The influence of the missing sub-grid scales can be modeled by the incorporation of a sub-grid scale model for particles. Two types of sub-grid scale models have been developed in the past. The first type is a stochastic model for the unresolved scales. The lack of the missing scales can be compensated in two manners. It can be indirectly compensated by adding broadband stochastic forcing to the momentum equation ${ }^{6}$ or directly by adding velocity differences to the particle equation of motion. ${ }^{7}$ The second type of sub-grid models is based on approximate deconvolution. ${ }^{8,9}$ The deconvolution of the filtered fluid velocity in LES allows to recover the energy up to the smallest resolved scales. ${ }^{10}$

Different procedures can be used for inverse filtering in order to substitute a defiltered fluid velocity in the particle equation of motion. In the dynamic eddy-viscosity model ${ }^{11}$ the filter appears only explicitly as a test filter and hence the defiltering method is rather arbitrary. ${ }^{12}$ In contrast, an explicit filter is used in the approximate deconvolution model (ADM $)^{13}$ and the same filter is used for the deconvolution of the fluid velocity in the fluid and particle equations. The sub-grid scale model for particles based on ADM was found to improve the prediction of statistical particle quantities substantially in channel flow at Reynolds numbers up to $\operatorname{Re}_{\tau}=395^{5,10,14,15}$ and in other turbulent flows. ${ }^{16-20}$ However, for channel flow at higher Reynolds numbers the improvement is less satisfactory. ${ }^{15}$

In 2012, Geurts and Kuerten ${ }^{21}$ conducted an investigation on ideal stochastic forcing of particles in LES of particle-laden channel flow in which the drag force exerted by the fluid on the particles was the only relevant force. The investigation was based on the results of DNS of particle-laden turbulent channel flow at several Reynolds numbers: $\operatorname{Re}_{\tau}=150,395$, and 950 and for different particle sizes corresponding to a range of Stokes numbers, i.e., particle relaxation time in wall units, $\mathrm{St}=0.2$, 1,5 , and 25 . They decomposed the fluid velocity $\boldsymbol{u}$ at the particle position, which is required in the particle equation of motion, into two contributions. The first is the deconvolution of the filtered fluid velocity, $\boldsymbol{u}^{*}$, at the particle position $\boldsymbol{x}$ and at time $t$ and is known in LES and the second is the residual velocity $\delta \boldsymbol{u}^{*}=\boldsymbol{u}(\boldsymbol{x}, t)-\boldsymbol{u}^{*}(\boldsymbol{x}, t)$, which needs to be modeled. Hence, the particle equation of motion can be written exactly as

$$
\frac{d \boldsymbol{v}}{d t}=\frac{\boldsymbol{u}^{*}(\boldsymbol{x}, t)-\boldsymbol{v}}{\tau_{p}}+\frac{\delta \boldsymbol{u}^{*}}{\tau_{p}}
$$

where $\boldsymbol{v}$ is the particle velocity and $\tau_{p}=\rho_{p} d_{p}^{2} /\left[\left(18 \rho_{f} v\right)\left(1+0.15 \mathrm{Re}_{p}^{0.687}\right)\right]$ is the particle relaxation time with $d_{p}$ is the particle diameter and $\rho_{p}$ is the particle mass density. Moreover, $\operatorname{Re}_{p}=d_{p} \mid \boldsymbol{u}(\boldsymbol{x}, t)$ $-v \mid / v$ is the particle Reynolds number and $v$ is the kinematic viscosity of the fluid. The first term on the right-hand side involves the well known particle sub-grid model based on approximate deconvolution investigated several times before. ${ }^{5,10,15}$ The second term requires additional modeling. Geurts and Kuerten ${ }^{21}$ showed that the statistical properties of $\delta \boldsymbol{u}^{*}$, like the root-mean square (RMS) of $\delta \boldsymbol{u}^{*}$, are basically identical for all investigated Stokes numbers, while the mean of $\delta \boldsymbol{u}^{*}$ is negligibly small. Moreover, the normalized probability density function and the time correlation function of $\delta \boldsymbol{u}^{*}$ hardly depend on Reynolds and Stokes numbers in the investigated range. This is a significant advantage for the development of a "stand alone" stochastic model for this term, which does not require explicit input from DNS but can be evaluated using LES quantities only.

The aim of this paper is the development of a sub-grid model that is a combination of ADM for the resolved scales and a stochastic model for the unresolved scales. The properties of the stochastic part of the model are based on the a priori DNS results at $\operatorname{Re}_{\tau}=950$. Hence, the model in its present formulation requires input from DNS. The model will be assessed by comparison with DNS results and with LES results without the stochastic part of the model.

The organization of this paper is as follows. In Sec. II, we describe the governing equations, the stochastic model for the particles and the numerical methods that are applied. We proceed in 
Sec. III with a presentation of the results of the proposed stochastic model; we identify the model parameters which lead to the most accurate correspondence of LES with DNS results. In Sec. IV, conclusions are presented.

\section{GOVERNING EQUATIONS AND NUMERICAL METHODS}

In this section, the governing equations for the fluid and for the particles are presented. Next, the hybrid stochastic-deconvolution sub-grid scale model added to the particle equation of motion is described. Then, the basic stochastic-deconvolution model is extended by adding the well-mixed property. Finally, the numerical methods are described.

The results of the $a$ priori $^{21}$ analysis are used to develop a sub-grid scale model for particles, which is a combination of ADM and a stochastic model. ADM is also used as the sub-grid scale model for the fluid. This model is tested on LES of incompressible particle-laden channel flow at Reynolds number $\operatorname{Re}_{\tau}=950$ based on the shear velocity $\boldsymbol{u}_{\tau}$ and half the channel height $H$. The fluid flow is solved with an Eulerian approach and the particles are treated in a Lagrangian way as point particles. The volume fraction of particles is sufficiently low for the one-way coupling regime to be valid. $^{2}$ This means that the effects of particles on the fluid flow and of particle-particle interaction are negligible.

The incompressible Navier-Stokes equation in rotational form reads

$$
\frac{\partial \boldsymbol{u}}{\partial t}+\omega \times \boldsymbol{u}+\nabla P=v \Delta \boldsymbol{u}+\boldsymbol{F},
$$

where $\boldsymbol{u}$ is the fluid velocity, $\omega=\nabla \times \boldsymbol{u}$ is the vorticity, $P=\frac{p}{\rho_{f}}+\frac{1}{2} \boldsymbol{u}^{2}$ with $p$ is the pressure relative to the linear mean pressure field which drives the flow, and $\rho_{f}$ is the fluid mass density. Furthermore, $\boldsymbol{F}$ is the driving force per unit mass in the streamwise direction parallel to the walls of the channel, constant in time and space. Since ADM is used as the sub-grid model for the fluid, the Navier-Stokes equation is solved in the following form: ${ }^{13}$

$$
\frac{\partial \overline{\boldsymbol{u}}}{\partial t}+\overline{\omega^{*} \times \boldsymbol{u}^{*}}+\nabla \bar{P}=v \Delta \overline{\boldsymbol{u}}+\boldsymbol{F}-\chi\left(I-Q_{N} G\right) \overline{\boldsymbol{u}},
$$

where

$$
u_{i}^{*}=Q_{N} \bar{u}_{i}=\sum_{k=0}^{N}(I-G)^{k} \bar{u}_{i}
$$

and $\bar{u}_{i}=G * u_{i}$ is the filtered velocity with filter kernel $G$. We set $N=5$ as advised by Stolz et al. ${ }^{13}$ The vorticity field of the deconvolved velocity is denoted by $\omega^{*}$. Moreover, in order to keep the kinetic energy contained in the smallest resolved scales constant in time, $\chi$ is dynamically adjusted. We adopt the same filter kernel and implementation of the model as originally proposed by Stolz et al. ${ }^{13}$ The filtered velocity field satisfies the continuity equation for incompressible flow:

$$
\nabla \cdot \overline{\boldsymbol{u}}=0 .
$$

The equations are non-dimensionalized with the shear velocity $\boldsymbol{u}_{\tau}$, half the channel height $H$, and the fluid mass density $\rho_{f}$. Time is non-dimensionalized with the fluid kinematic viscosity $v$ and $\boldsymbol{u}_{\tau}$.

Lagrangian tracking of particles is performed by numerically integrating the equation:

$$
\frac{d x(t)}{d t}=\boldsymbol{v},
$$

together with Eq. (1). Note that in LES the particle Reynolds number is based on the defiltered fluid velocity at the particle position. A solution of the Langevin equation

$$
\frac{d \delta u_{i}^{*}}{d t}=-\frac{\delta u_{i}^{*}}{\tau}+C_{i} w_{i}(t)
$$

is taken as the model for the stochastic part in Eq. (1). ${ }^{7,22}$ Here, $\tau$ is the Lagrangian correlation time of $\delta \boldsymbol{u}^{*}, C_{i}=\delta u_{i, r m s}^{*} \sqrt{2 / \tau}$ and $w_{i}(t)$ is Gaussian white noise of unit variance. Formally, 
$w_{i}(t)=d W_{i}(t) / d t$, where $W_{i}(t)$ stands for a Wiener process. The RMS of $\delta \boldsymbol{u}^{*}$ and the correlation time both follow from the a priori analysis. ${ }^{21}$ The profiles of these a priori quantities are averaged over the streamwise and spanwise directions and time and are hence only functions of the wall-normal coordinate.

It can be shown that Eq. (7) cannot be correct for inhomogeneous turbulent flow and particles with small Stokes number. To this end, we take the Eulerian average of the wall-normal component of this equation at a fixed wall-normal coordinate. Since the mean of $w(t)$ equals zero and according to the a priori results also the mean of $\delta \boldsymbol{u}^{*}$ equals zero, the mean of the right-hand side equals zero. On the other hand, the mean of the left-hand side can for passive particles be written as

$$
\left\langle\frac{d}{d t} \delta u_{y}^{*}\right\rangle=\frac{\partial}{\partial t}\left\langle\delta u_{y}^{*}\right\rangle+\left\langle\boldsymbol{u} \cdot \nabla \delta u_{y}^{*}\right\rangle .
$$

Since the mean of $\delta \boldsymbol{u}^{*}$ equals zero, the first term on the right-hand side vanishes. The second term can be written as $\frac{d}{d y}\left\langle u_{y} \delta u_{y}^{*}\right\rangle$, where the fact that the velocity field is divergence free has been used. The a priori results show that this term is unequal to zero for the present inhomogeneous flow. Since the right-hand side of Eq. (7) equals zero, while the left-hand side does not, an extra term is needed in Eq. (7).

The missing term in Eq. (7), which is relevant in inhomogeneous turbulence, is related to the well-mixed condition, which is an important property for statistical particle models. This condition requires that passive particles, which initially are uniformly distributed over the computational domain, should retain this uniform distribution at later times. The basic hybrid stochastic-deconvolution model presented above does not satisfy the well-mixed condition because passive particles tend to go to the walls. Therefore, the following extension of the basic hybrid stochastic-deconvolution model is proposed. The well-mixed condition can be satisfied in a similar way as proposed by Thomson ${ }^{23}$ for the Langevin equation governing dispersion of passive particles in inhomogeneous turbulent flow. It leads to an extra term in the stochastic equation for the wall-normal component of $\delta u_{y}^{*}$ :

$$
\frac{d \delta u_{y}^{*}}{d t}=-\frac{\delta u_{y}^{*}}{\tau}+C w(t)+f(\mathrm{St}) \frac{d\left(\sigma_{y}^{*}\right)^{2}}{d y}\left(1+\frac{1}{2} \frac{\left(\delta u_{y}^{*}\right)^{2}}{\left(\sigma_{y}^{*}\right)^{2}}\right),
$$

where $y$ is the wall-normal coordinate and $\sigma_{y}^{*}$ is the RMS of $\delta u_{y}^{*}$ obtained from the $a$ priori results. ${ }^{21}$

For particles with higher Stokes number, the argument given above regarding the need to complete the model expressed in Eq. (7) with the missing term cannot be applied. These particles move with velocity $\boldsymbol{v}$ instead of $\boldsymbol{u}$ and the correlation between $\boldsymbol{v}$ and $\boldsymbol{u}$ decreases with increasing Stokes number and tends to zero for large Stokes number, since the particle and fluid velocities become decorrelated in this limit for very large particles. Moreover, we applied the continuity equation for the fluid velocity to the left-hand side of Eq. (8), which is not possible for inertial particles. In order to accommodate this dependence on Stokes number, the well-mixed term is multiplied with a weight-factor, which is taken as a function of the Stokes number $f(\mathrm{St})$. This function should be between 0 and 1 . In order to satisfy the well-mixed condition for passive particles, $f(\mathrm{St}=0)$ should be equal to 1 and, as argued above, the other limit is $f(\mathrm{St} \rightarrow \infty)=0$.

The function $f(\mathrm{St})$ can be estimated from the a priori results. As shown above, it is related to the term $\left\langle\boldsymbol{v} \cdot \nabla \delta u_{y}^{*}\right\rangle$. Figure 1 shows this term in wall units as a function of the wall-normal coordinate for several values of the Stokes number. For larger values of the Stokes number than shown in the figure, the results become less accurate, since the particle concentration at some distance from the wall rapidly decreases because of turbophoresis. The spatial derivative inside the average in this term suffers more from this effect than first and second order moments of $\delta \boldsymbol{u}^{*}$. The results shown in Fig. 1 exhibit a decrease of the maximum of $\left\langle\boldsymbol{v} \cdot \nabla \delta u_{y}^{*}\right\rangle$ with Stokes number. In fact, this decrease closely follows an exponential dependence on St, as will be shown later.

Next, we proceed with a description of the numerical methods applied to solve the system of equations for fluid and particles. The turbulent channel flow is simulated with the use of periodic conditions in the streamwise and spanwise directions and no-slip conditions at the walls. Therefore, the use of a pseudo-spectral method is convenient. ${ }^{10}$ In the two periodic directions a Fourier-Galerkin method is applied and a Chebyshev-collocation method is used in the wall-normal direction. The 


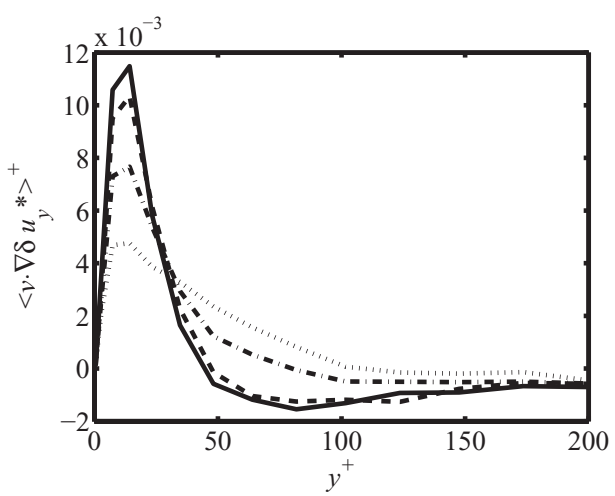

FIG. 1. A priori correlation $\left\langle\boldsymbol{v} \cdot \nabla \delta u_{y}^{*}\right\rangle$ in wall units as a function of the wall-normal coordinate in wall units for $\operatorname{Re}_{\tau}=950$; solid: $\mathrm{St}=0.2$, dashed: $\mathrm{St}=0.4$, dashed-dotted: $\mathrm{St}=1$, dotted: $\mathrm{St}=2$.

continuity equation is satisfied up to machine accuracy by the use of the influence matrix method. ${ }^{24}$ A second-order accurate method is used for time integration. It is a combination of an explicit three-stage Runge-Kutta method and the implicit Crank-Nicolson method. The viscous terms and the pressure are treated with the implicit method, whereas the convective and sub-grid scale terms are integrated explicitly in time. For the calculation of the nonlinear terms, including the sub-grid scale terms, aliasing is prevented by the application of the $3 / 2$ rule. The deconvolution necessary in the calculation of the sub-grid model can easily be performed in spectral space in the two periodic directions. ${ }^{10}$ In the wall-normal direction it requires a matrix-vector multiplication.

The equations of motion for the particles are integrated in time with a second-order accurate two-stage Runge-Kutta method. For the interpolation of the fluid velocity to the particle positions a fourth-order accurate method is applied. It consists of Lagrangian interpolation in the two periodic directions and Hermite interpolation in the wall-normal direction. It is known that the order of this interpolation is important on relatively coarse LES grids.${ }^{10}$ Compared to fourth-order interpolation, second-order interpolation acts as an additional filter, which counteracts the deconvolution operator. ${ }^{10}$ Particles collide elastically with the walls of the channel. If a particle leaves the computational domain through one of the periodic boundaries, it is re-inserted at the other side with the same properties.

The stochastic equation (7) is treated in the following way. Once every time step the new value of $\delta \boldsymbol{u}^{*}$ is determined by generating a new value for the random variable $\boldsymbol{w}$ and subsequently using the exact solution to the ordinary differential equation with coefficients $C$ and $\tau$ frozen at the values of the current particle position. ${ }^{25}$ The values of the correlation time and a priori RMS of $\delta \boldsymbol{u}^{*}$ are stored in the grid points in the wall-normal direction and are linearly interpolated to the position of the particle. Since these quantities are smooth functions of the wall-normal coordinate, this type of interpolation appears sufficiently accurate. The nonlinear term on the right-hand side of Eq. (9) is treated with the Euler forward method.

The sizes of the computational domain are $2 \pi H, \pi H$, and $2 H$ in the streamwise, spanwise, and wall-normal direction, respectively. The corresponding numbers of grid nodes are 96, 128, and 97. With the resulting grid spacings the requirements for resolved LES $^{26}$ are satisfied, while a significant reduction in resolution is achieved compared to the DNS resolution of $768 \times 768 \times 385$ that was adopted at $\operatorname{Re}_{\tau}=950$. In particular, in the LES $\Delta x^{+}=62, \Delta z^{+}=23$, while $\Delta y^{+}$ranges between 0.5 close to the wall and 30 at the center of the channel. The size of the domain in streamwise and spanwise direction is much smaller than in the DNS at the same Reynolds number by Hoyas and Jiménez. ${ }^{27}$ However, our DNS results for first and second order moments of the fluid velocity agree well with their results and the two-point correlations of all three velocity components tend to zero within half the length of the channel.

The initial state of the particles is taken from a random uniform distribution for the position and a particle velocity equal to the fluid velocity at the particle position. The initial value of $\delta \boldsymbol{u}^{*}$ is chosen randomly from a Gaussian distribution with the same RMS as obtained in the a priori results. Twelve sizes of particles are considered in this study. The diameters of the particles are chosen in 


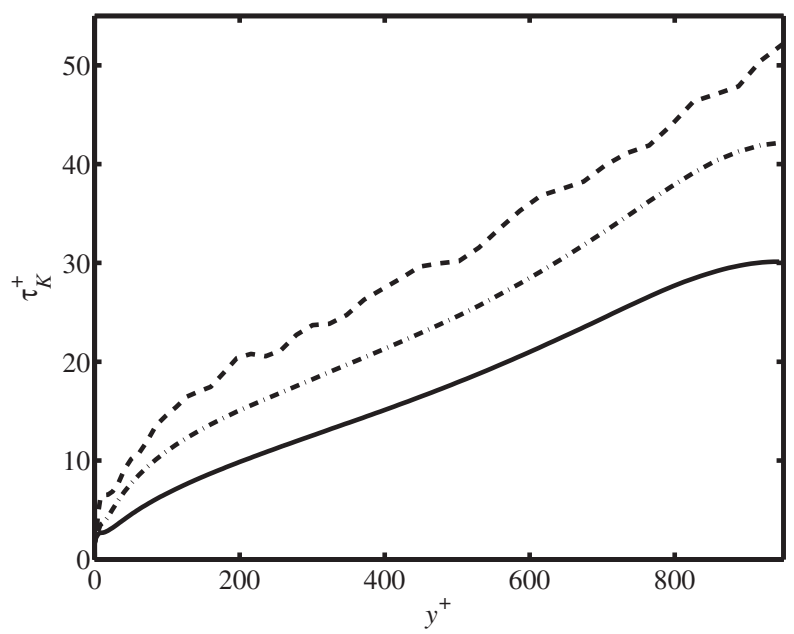

FIG. 2. Kolmogorov time, resolved Kolmogorov time scale in LES and a priori Lagrangian correlation time $\tau$ in wall units as a function of the wall-normal coordinate in wall units for $\mathrm{Re}_{\tau}=950$; solid: Kolmogorov time, dashed-dotted: LES-ADM resolved Kolmogorov time scale, dashed: a priori $\tau$ results and $\mathrm{St}=1$.

such a way that the corresponding Stokes numbers are equal to $0.2,0.4,0.7,1,1.5,2,3,4,5,6,8$, and 25. For each size, 64000 particles are tracked. Statistical results of the particles are averaged over the two homogeneous directions and over time in the statistically steady state and presented as functions of the wall-normal coordinate. To this end the wall-normal direction is divided in 128 bins, according to a Chebyshev distribution, and statistics are collected on this grid.

\section{RESULTS}

In this section, we first present results obtained with the basic stochastic-deconvolution model. These results show significant deviations from DNS in case of particles with low Stokes numbers, which can be addressed by extending this basic model with the well-mixed correction term. Subsequently, results of the extended stochastic model are presented and analyzed.

In Fig. 2, the correlation time $\tau$, obtained from the a priori results, is compared with typical time scales of the flow in DNS and LES. For DNS the Kolmogorov time $\tau_{K}=\sqrt{v / \epsilon}$, where $\epsilon$ is the dissipation rate of the turbulent kinetic energy, is considered as the typical time scale. For LES this time scale, with $\epsilon$ evaluated using the resolved scales only, is selected; this will be referred to as the resolved Kolmogorov time. The correlation time shown in the figure is for the streamwise component of $\delta \boldsymbol{u}^{*}$ and for $\mathrm{St}=1$. The results for the other components and Stokes numbers are very similar. The figure shows that the typical time scale of LES is larger than that of DNS. At the resolution and spatial filter-width selected the difference is below $30 \%$. The rather modest differences in these time scales are indicative of the fact that the LES is indeed well resolved. The dependence of the correlation time of $\delta \boldsymbol{u}^{*}$ on the wall-normal coordinate is very similar to that of the typical time scale of the LES and is larger by a factor of only about 1.2. Because of this close agreement we expect that $\tau$ can be well predicted based on the LES results only, resulting in a further step toward a model that is based entirely on information that is available during an LES.

Figure 3 shows the RMS of the wall-normal component of $\delta \boldsymbol{u}^{*}$ as a function of the wall-normal coordinate in wall units $y^{+}$. The differences between the a priori results for the four different Stokes numbers are small. This limited dependence of $\delta \boldsymbol{u}^{*}$ on Stokes number was reported by Geurts and Kuerten. ${ }^{21}$ Since the flow field is independent of Stokes number, in view of the one-way coupling adopted here, the dependence of $\delta \boldsymbol{u}^{*}$ on Stokes number can only be caused by preferential concentration. For increasing Stokes number the RMS first decreases slightly until $\mathrm{St}=5$, which corresponds to the case where the particle relaxation time is approximately equal to the Kolmogorov time averaged over the wall-normal direction, and then increases again. This implies that the effect of preferential concentration of particles on this quantity is strongest for $\mathrm{St}=5$. On the other hand, 


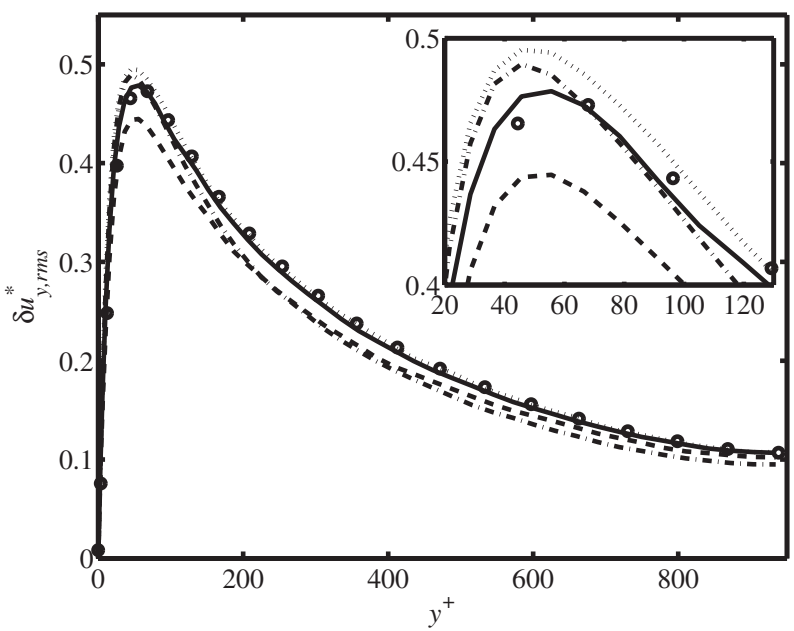

FIG. 3. The RMS of $\delta u_{y}^{*}$ as a function of the wall-normal coordinate in wall units for $\operatorname{Re}_{\tau}=950$; dotted: a priori $\mathrm{St}=0.2$, solid: a priori $\mathrm{St}=1$, dashed: a priori $\mathrm{St}=5$, dashed-dotted: a priori $\mathrm{St}=25, \circ$ : a posteriori $\mathrm{St}=1$ with weighting factor $f(1)=0.6$.

similar as at lower Reynolds numbers, the effect of preferential concentration on the RMS of the wall-normal component of the fluid velocity evaluated at the particle position is largest for larger Stokes numbers. ${ }^{28}$ This implies that the preferential concentration for large particles is mainly due to the larger scales of the velocity field, which are absent in $\delta \boldsymbol{u}^{*}$. Figure 3 shows a good agreement between a priori and a posteriori results for the RMS of $\delta u_{y}^{*}$. We infer that the hybrid stochasticdeconvolution model shows the correct statistical results for $\delta \boldsymbol{u}^{*}$. Note that the results shown in Fig. 3 do not change if the well-mixed term is not taken into account.

Almost all statistical particle properties appeared quite insensitive to small variations in $\delta \boldsymbol{u}_{r m s}^{*}$ caused by varying the Stokes number. These variations, however, do result in deviations in the mean relative (particle minus fluid velocity) wall-normal velocity component up to $20 \%$, which accumulate in some discrepancies in the particle concentration due to turbophoresis close to the wall in the long-time regime. Therefore, we used the a priori results for each Stokes number in this paper.

In general, all statistical particle velocity properties such as the mean and the RMS of the three velocity components are similar or slightly improved by the use of the hybrid stochasticdeconvolution model (Eq. (7)) in comparison to the results of the sub-grid model for the particles with only the approximate deconvolution model in the particle equation of motion. For the biggest particles considered here $(\mathrm{St}=25)$ also the prediction of the particle concentration is accurate. However, the turbophoresis effect is strongly overpredicted for smaller particles, especially for particles with $\mathrm{St} \leq 1$.

The reason for the overprediction of the particles concentration close to the wall is the fact that the stochastic model does not satisfy the well-mixed condition. ${ }^{23}$ Indeed, for passive particles, which follow the fluid flow exactly, the use of the stochastic model to generate the unfiltered fluid velocity at the particle position results in a continuing increase in particle concentration near the walls. For inertial particles with small Stokes numbers a similar behavior is found, in contrast with the DNS results, where a steady particle concentration is obtained after some time.

Since the particle concentration is the most critical quantity, we will first turn our attention to that, and later consider particle velocity statistics. The concentration results of the smallest particles considered do show very good agreement with the DNS results provided the well-mixed term is added to the basic hybrid stochastic-deconvolution model (9) using $f(0.2) \approx 1$. The effect of the well-mixed term is to decrease the particle concentration close to the walls. We find that only for passive particles and inertial particles with St $\ll 1$ the prediction of the particle concentration close to the walls is in close agreement with DNS results. The situation is different in case of somewhat larger particles. Figure 4 shows the particle concentration close to the walls for particles with $\mathrm{St}=1$ 


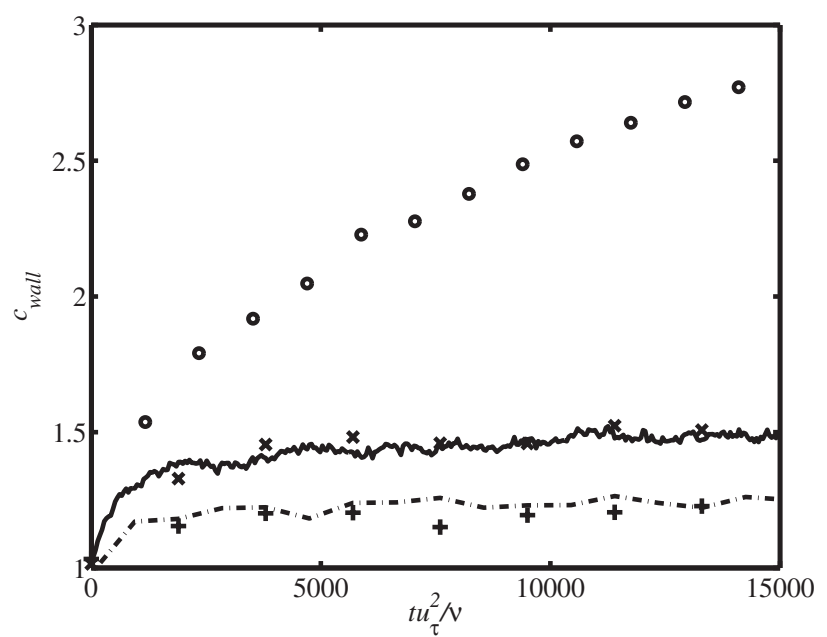

FIG. 4. The particle concentration close to the walls as a function of time in wall units, $t^{+}$, for $\mathrm{St}=1$; solid: DNS, dasheddotted: LES with ADM for particles, $\circ$ : LES with ADM and stochastic model for particles, +: LES with ADM and stochastic model with the well-mixed term with $f(1)=1.0$ for particles, $\times$ : LES with ADM and stochastic model with $f(1)=0.6$ for particles.

as a function of time in wall units $t^{+}=t u_{\tau}{ }^{2} / \nu$. In particular, the channel is uniformly divided into 40 bins and only the concentration of particles in the first bin at both walls is shown.

We observe that with full inclusion of the well-mixed term the decrease of the concentration close to the walls is too large as was observed for particles with St $>0.2$ and illustrated explicitly in Fig. 4 for particles with $\mathrm{St}=1$. This underestimation was found to increase with increasing Stokes number. It appears that the well-mixed term is only required for small particles and should be reduced in magnitude for particles with significant inertia. We proposed to multiply the well-mixed term by a function of Stokes number, $f(\mathrm{St})$, which equals 1 for $\mathrm{St}=0$ and monotonically decreases to zero. In Fig. 4 , it can be seen that very good agreement with the DNS results is obtained if $f(1)=0.6$. In Fig. 4, also the results with only ADM are included. Similar to the results with the well-mixed term with $f(1)=1$, they show an underprediction of turbophoresis.

Figure 5 shows the particle concentration close to the walls as a function of non-dimensionalized time $t^{+}$for $\mathrm{St}=4$ for the hybrid stochastic-deconvolution model for several values of $f(4)$. The figure also includes the result if only ADM is applied as the sub-grid model in the particle equation of motion. The figure clearly shows that incorporation of the stochastic part of the model improves the prediction of the particle concentration close to the walls. However, the time history of the particle concentration appears not predicted correctly for any value of $f(4)$. Therefore, we chose the value which gives the best agreement in the statistically steady state in which the particle concentration does not increase further, but only fluctuates in time. Since this steady state is not reached yet at this Stokes number, we extrapolated the results for the particle concentration in time, incorporating the observed exponential dependence of the concentration close to the wall on time. For $\mathrm{St}=4$ we find in this way $f(4)=0.15$ as optimal value, which is not very sensitive to the chosen extrapolation method. The prediction of this quantity for $\mathrm{St}=4$ is the worst amongst all Stokes numbers considered. Moreover, the particle concentration close to the wall is the most sensitive quantity. However, the wall-normal concentration profile is reasonably well predicted also in the transient state.

In a similar way optimal values of $f(\mathrm{St})$ have been found for the whole range of Stokes numbers considered in this paper. The results of this optimization can be seen in Fig. 6. These results are well described by $f(\mathrm{St})=\exp (-\mathrm{St} / 2)$, which is in remarkable agreement with the dependence of the maximum of the correlation $\left\langle\boldsymbol{v} \cdot \nabla \delta u_{y}^{*}\right\rangle$ on Stokes number shown in Fig. 1.

The wall-normal concentration profiles in the steady state for $\mathrm{St}=1$ and 5 are presented in Figs. 7 and 8 . The results are averaged over a time interval of $2 \times 10^{4}$ in wall units during the steady state. The figures show that also the concentration profile in the steady state is well predicted by the 


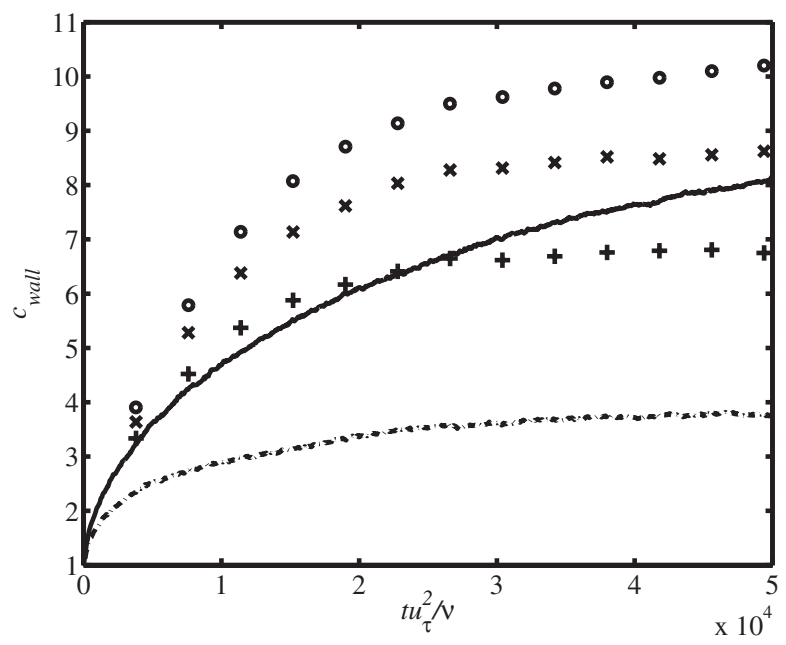

FIG. 5. The particle concentration close to the walls as a function of time in wall units, $t^{+}$, for $\mathrm{St}=4$; solid: DNS, dasheddotted: LES with ADM for particles, LES with ADM and stochastic model for particles $\circ$ : with $f(4)=0.05, \times$ : with $f(4)=0.15,+$ : with $f(4)=0.3$.

new model. Moreover, the inclusion of the stochastic part of the model significantly improves the prediction of this quantity compared to the results of the ADM alone, which are also included in the figures.

Next, the effect of the stochastic model on particle velocity properties will be shown. The phenomenon of turbophoresis is caused by the mean relative particle velocity in the wall-normal direction relative to the fluid velocity. In Figs. 9 and 10, the mean relative particle velocity is presented as a function of the distance from the wall in wall units, $y^{+}$, for $\mathrm{St}=1$ and 5 , respectively. The figures show that for $\mathrm{St}=1$ the mean relative velocity is too small compared to the DNS results in the near-wall region if only the approximate deconvolution model is applied in the particle equation of motion. This leads to the under-prediction of the particle concentration close to the walls. The addition of the stochastic model in the particle equation of motion that includes the weighted well-mixed term results in a significant improvement in the agreement with the DNS results. For $\mathrm{St}=5$ the inclusion of the stochastic model slightly deteriorates the prediction of the mean relative wall-normal velocity.

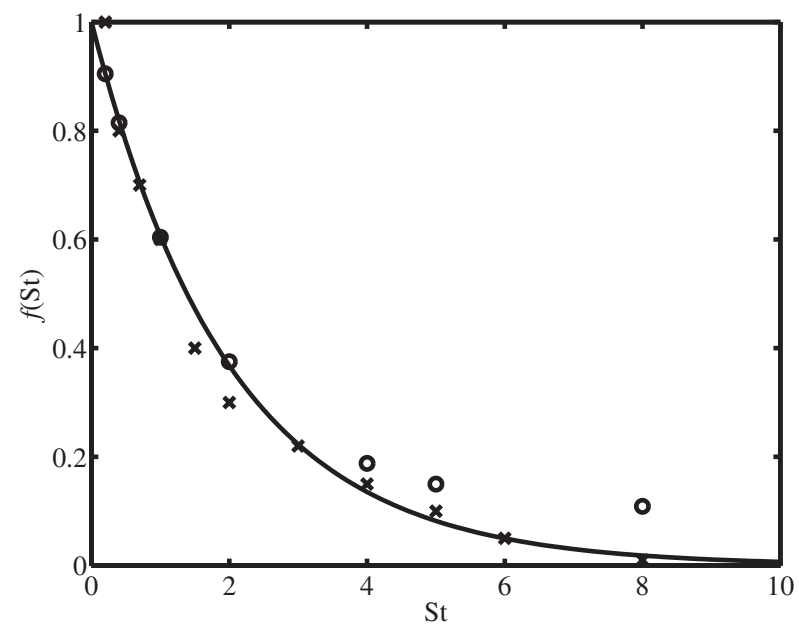

FIG. 6. The optimal values of $f(\mathrm{St})$ (crosses) and a general trend (solid) given by $f(\mathrm{St})=\exp (-\mathrm{St} / 2)$. Included are also the scaled maxima of the term $\left\langle\boldsymbol{v} \cdot \nabla \delta u_{y}^{*}\right\rangle$ shown in Fig. 1 (circles). 


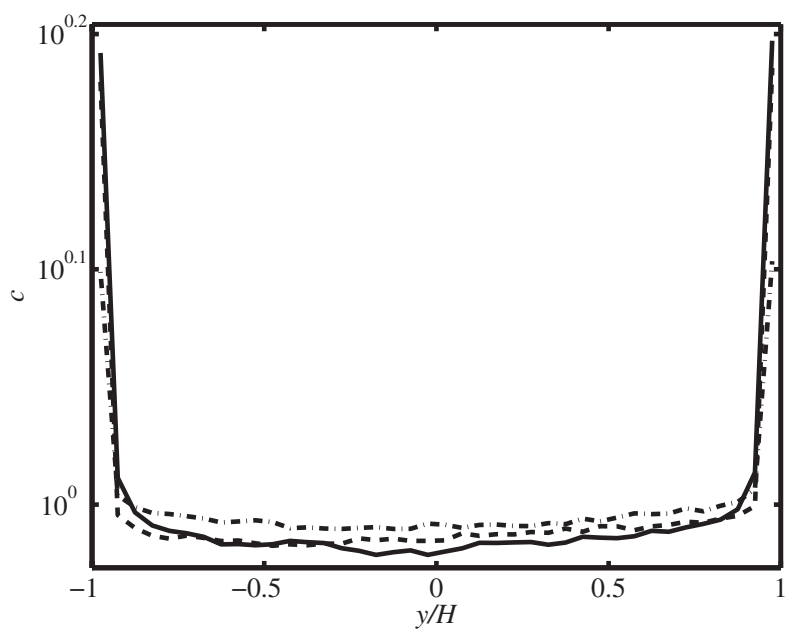

FIG. 7. The steady-state particle concentration profile in the wall-normal direction for $\mathrm{St}=1$; solid: DNS, dashed-dotted: LES with ADM for particles, dashed: LES with ADM and stochastic model with $f(1)=0.6$ for particles.

Figure 11 presents the mean relative streamwise particle velocity as a function of the wallnormal coordinate in wall units for $\mathrm{St}=1$. The results of the new model are almost the same as the results of the model without the stochastic part.

Figure 12 (left) shows the RMS of the streamwise particle velocity component as a function of the wall-normal coordinate in wall units, $y^{+}$, for $\mathrm{St}=1$. The stochastic model has almost no influence on the RMS of this component of the particle velocity. The mean streamwise particle velocity profile is basically identical for simulations with and without the stochastic model. However, the stochastic model improves the RMS of the wall-normal and spanwise particle velocity components. The RMS of the wall-normal particle velocity component can be seen in Fig. 12 (right), which presents this quantity as a function of the distance from the wall in wall units, $y^{+}$, for $\mathrm{St}=1$. The RMS of the spanwise particle velocity component can be seen in Fig. 12 (bottom). The presence of the wellmixed term does not affect the results for the streamwise and spanwise particle velocity components and the influence on the wall-normal component is barely visible.

The results for other Stokes numbers are very similar. Figure 13 shows the RMS of the spanwise particle velocity as a function of the wall-normal coordinate in wall units for $\mathrm{St}=5$. A similar

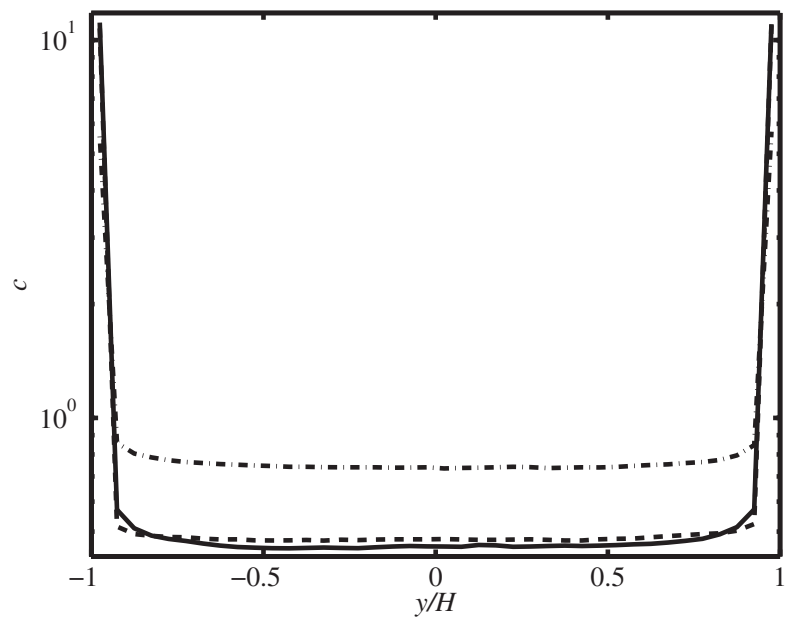

FIG. 8. The steady-state particle concentration profile in the wall-normal direction for $\mathrm{St}=5$; solid: DNS, dashed-dotted: LES with ADM for particles, dashed: LES with ADM and stochastic model with $f(5)=0.1$ for particles. 


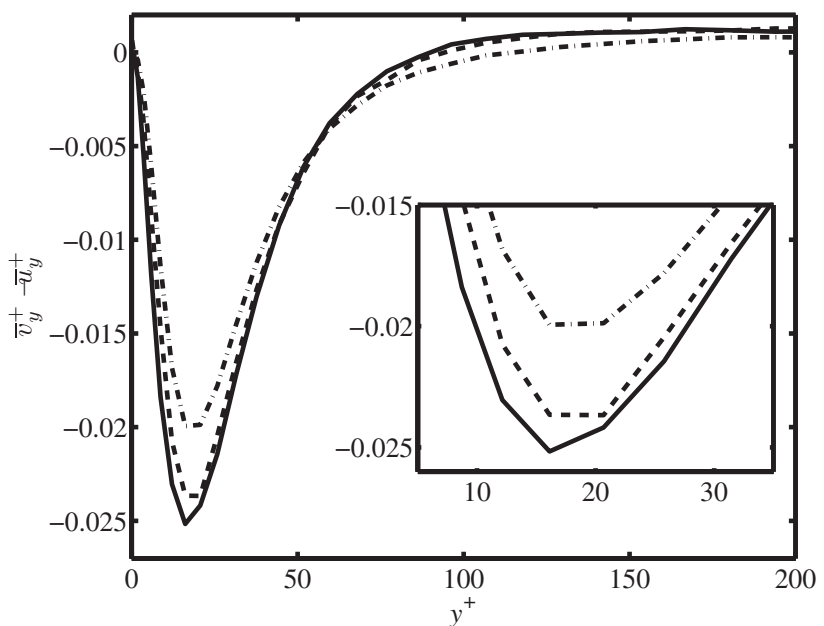

FIG. 9. The mean relative wall-normal particle velocity as a function of the wall-normal coordinate in wall units for St $=1$; solid: DNS, dashed-dotted: LES with ADM for particles, dashed: LES with ADM and stochastic model with $f(1)=0.6$ for particles.

improvement in the prediction of this quantity is obtained when the new model is applied as for $\mathrm{St}=1$. Generally, in all cases the hybrid stochastic-deconvolution model shows a better agreement with DNS results for the wall-normal and spanwise particle velocity fluctuations compared to simulations in which only the approximate deconvolution model is applied in the particle equation of motion. The results for the streamwise particle velocity fluctuations are hardly affected by the stochastic contribution to the sub-grid model.

In Fig. 14 (right), the probability density function (PDF) of the wall-normal component of $\delta \boldsymbol{u}^{*}$ for $\mathrm{St}=1$ is shown. The PDF depends on the wall-normal coordinate of the particles and the figure shows the result for particles in a small region around $y^{+}=60$, which is close to the maximum of the RMS of $\delta u_{y}^{*}$. Deviations in the PDF will result in the largest differences in this region. Although the turbulence is inhomogeneous, the PDF turns out to be Gaussian. This is a consequence of the use of Gaussian white noise in the stochastic equation for $\delta \boldsymbol{u}^{*}$. In contrast, the a priori results display a slightly higher probability for large deviations from the mean value of $\delta u_{y}^{*}$ and a higher peak value

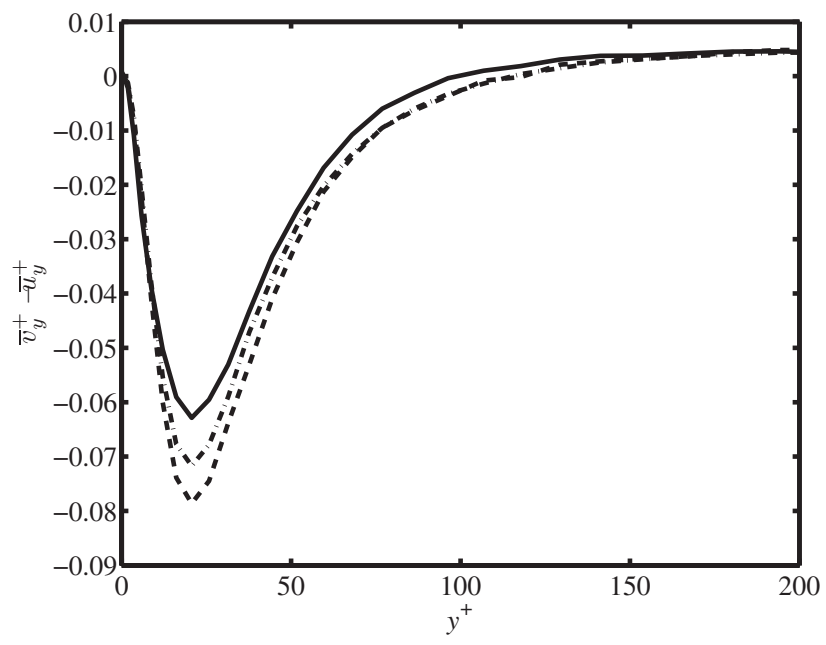

FIG. 10. The mean relative wall-normal particle velocity as a function of the wall-normal coordinate in wall units for $\mathrm{St}=5$; solid: DNS, dashed-dotted: LES with ADM for particles, dashed: LES with ADM and stochastic model with $f(5)=0.1$ for particles. 


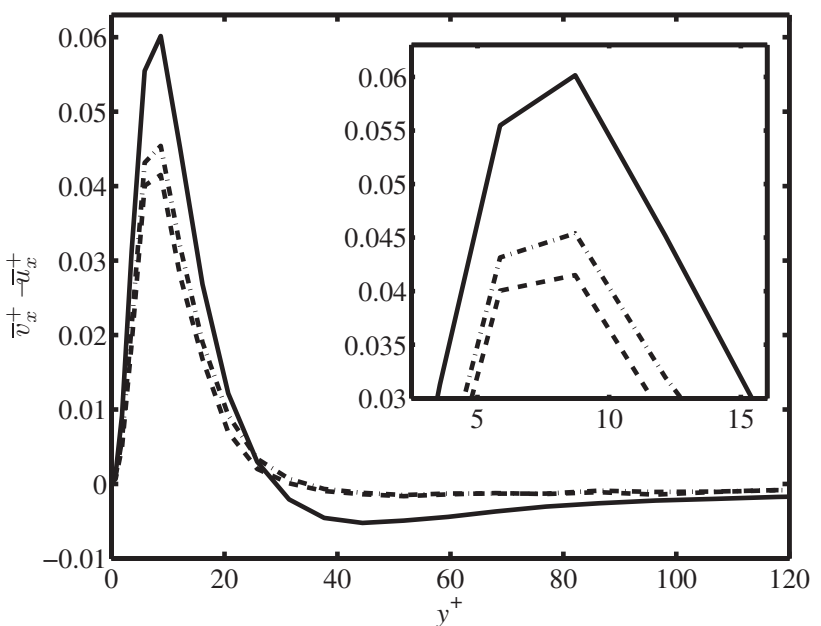

FIG. 11. The mean relative streamwise particle velocity as a function of the wall-normal coordinate in wall units for $\mathrm{St}=1$; solid: DNS, dashed-dotted: LES with ADM for particles, dashed: LES with ADM and stochastic model with $f(1)=0.6$ for particles.
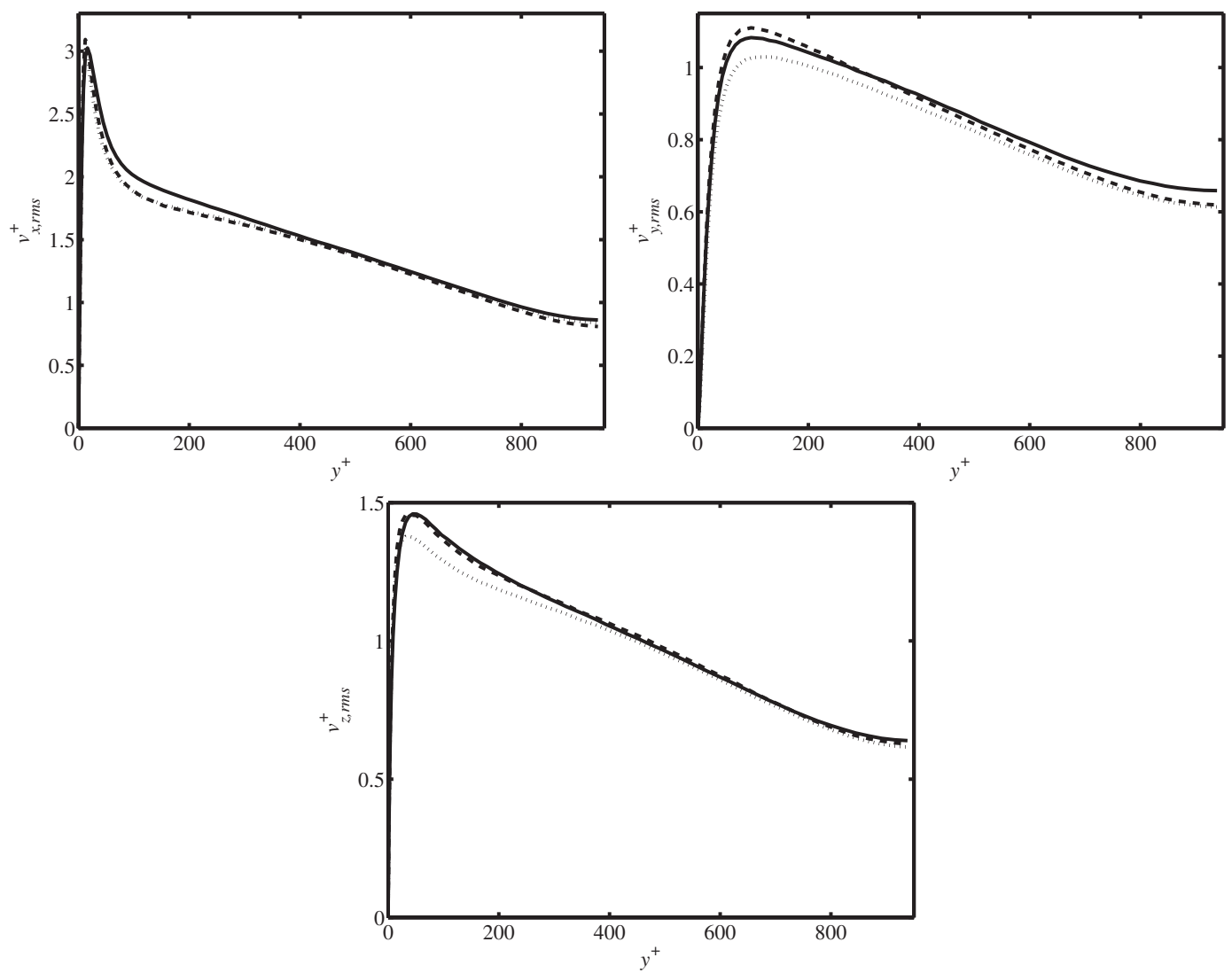

FIG. 12. The RMS of the streamwise (left), wall-normal (right), and spanwise (bottom) particle velocity as a function of the wall-normal coordinate in wall units for $\mathrm{St}=1$; solid: DNS, dashed-dotted: LES with ADM for particles, dashed: LES with ADM and stochastic model with $f(1)=0.6$ for particles. 


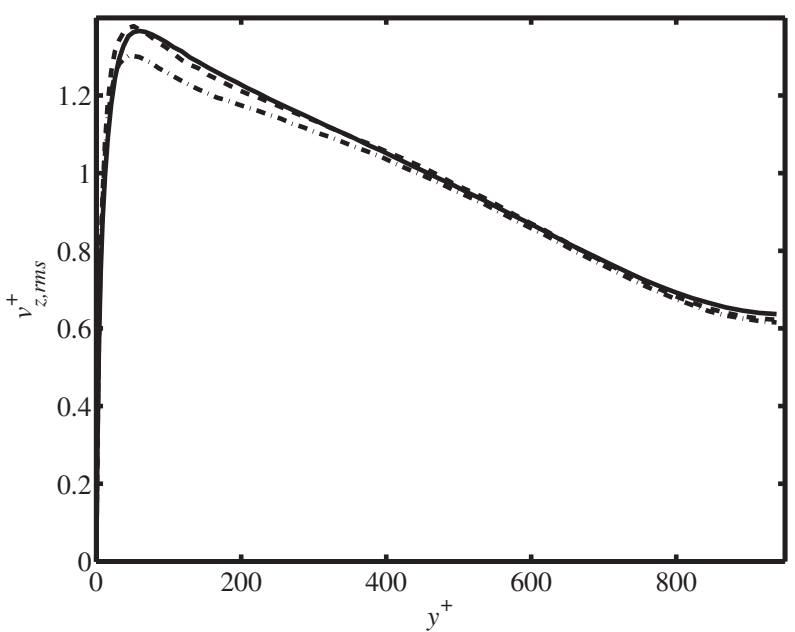

FIG. 13. The RMS of the spanwise particle velocity as a function of the wall-normal coordinate in wall units for $\mathrm{St}=5$; solid: DNS, dashed-dotted: LES with ADM for particles, dashed: LES with ADM and stochastic model with $f(5)=0.1$ for particles.
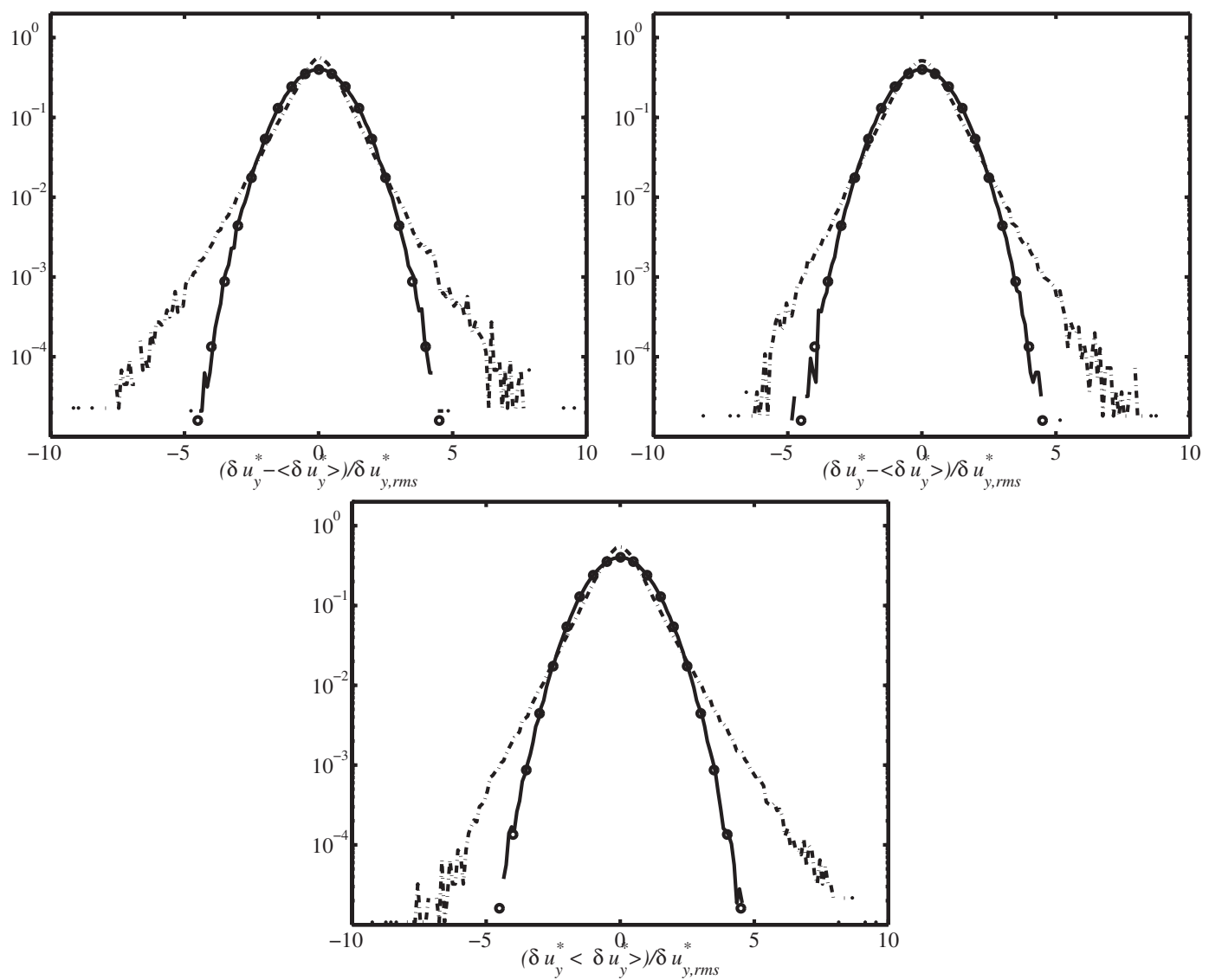

FIG. 14. PDF of $\delta u_{y}^{*}$ at $y^{+}=30$ (left), $y^{+}=60$ (right), and $y^{+}=200$ (bottom) for St $=1$; solid: LES, dashed-dotted: a priori DNS, circles: Gaussian distribution. 
at deviation zero. This suggests a somewhat different intermittent behavior of the LES dynamics, compared to the DNS findings. Note, however, that for the sub-grid model based on ADM only $\delta \boldsymbol{u}^{*}$ will be equal to zero, resulting in a delta-function for the PDF. Therefore, the hybrid stochasticdeconvolution model is an improvement over the model based on ADM only. Similar results for the PDF are obtained at different wall-normal positions, as can be seen in Fig. 14, which also shows the PDFs at $y^{+}=30$ (left) and $y^{+}=200$ (bottom).

\section{CONCLUSIONS AND DISCUSSION}

We developed a hybrid stochastic-deconvolution model for the particle equation of motion in LES of particle-laden turbulent flow. The coefficients in the stochastic part of the model, $\delta u_{r m s}^{*}$ and the Lagrangian correlation time $\tau$, are based on a priori results of DNS of the same flow. The correlation time $\tau$ appears quite independent of Stokes number, while $\delta u_{r m s}^{*}$ slightly varies with Stokes numbers. Almost all LES particle results are independent of these slight variations and can be predicted well on the basis of the Stokes number independent stochastic model. The mean relative wall-normal velocity component is also accurately captured by the model. This velocity component is crucial for the prediction of turbophoresis. Since turbophoresis arises from the accumulation of the small wall-normal particle motion over a very long time, it is more strongly dependent on the precise choice of the coefficients. Therefore, the small dependence of the a priori results on Stokes number has nevertheless been taken into account to obtain good agreement with DNS results for all particle sizes.

For particles with small Stokes number an important requirement is that the stochastic model satisfies the well-mixed condition. Conversely, the inclusion of the standard well-mixed term in the model was found to deteriorate the results for larger particles. Therefore, a Stokes-number dependent well-mixed term has been proposed. The Stokes-number dependency can be well described by the function $f(\mathrm{St})=\exp (-\mathrm{St} / 2)$, as was obtained from a direct comparison with DNS results over a range of Stokes numbers. This approximately exponential decay is also observed in a priori results of $\left\langle\boldsymbol{v} \cdot \nabla \delta u_{y}^{*}\right\rangle$.

In the present model, a Gaussian white noise term has been applied. In the future it will be investigated whether the application of non-Gaussian white noise will lead to further improvement of the model. Moreover, it will be studied whether the model coefficients that are now obtained from the a priori results can also be found from LES alone, leading to a particle model that is fully obtained without reference to DNS input. The close resemblance between the Lagrangian correlation time $\tau$ and the resolved Kolmogorov time might be useful in this respect.

For higher particle volume concentrations than considered in this paper, two-way coupling between particles and fluid becomes increasingly important. The sub-grid model proposed in this paper can equally be applied in case of two-way coupling, by using the conservation principle that the drag force exerted by the fluid on a particle is exactly opposite to the reaction force by the particle on the fluid. In this way the stochastic contribution to the drag force also enters the two-way coupling force on the fluid.

\section{ACKNOWLEDGMENTS}

This research is supported by the Dutch Technology Foundation STW, applied-science division of NWO (Netherlands Organization for Scientific Research), and the Technology Program of the Ministry of Economic Affairs of the Netherlands. This work was sponsored by the Stichting Nationale Computerfaciliteiten (National Computing Facilities Foundation, NCF) for the use of supercomputer facilities, with financial support from the Nederlandse Organisatie voor Wetenschappelijk Onderzoek, NWO. We thank the DEISA Consortium (www.deisa.eu), co-funded through the EU FP6 project RI-031513 and the FP7 project RI-222919, for support within the DEISA Extreme Computing Initiative. 
${ }^{1}$ B. Bunner and G. Tryggvason, "Direct numerical simulations of three-dimensional bubbly flows," Phys. Fluids 11, 19671969 (1999)

${ }^{2}$ S. Elghobashi, "On predicting particle-laden turbulent flows," Appl. Sci. Res. 52, 309-329 (1994).

${ }^{3}$ B. J. Geurts, Elements of Direct and Large-Eddy Simulation (Edwards Publishing, 2003).

${ }^{4}$ W. S. J. Uijttewaal and R. V. A. Oliemans, "Particle dispersion and deposition in direct numerical and large eddy simulation of vertical pipe flows," Phys. Fluids 8, 2590-2604 (1996).

${ }^{5}$ J. G. M. Kuerten and A. W. Vreman, "Can turbophoresis be predicted by large-eddy simulation?," Phys. Fluids 17, 011701 (2005).

${ }^{6}$ A. K. Kuczaj and B. J. Geurts, “Mixing in manipulated turbulence,” J. Turbul. 7, N67 (2006).

${ }^{7}$ O. Simonin, E. Deutsch, and J. P. Minier, "Eulerian prediction of the fluid/particle correlated motion in turbulent two-phase flows," Appl. Sci. Res. 51, 275-283 (1993).

${ }^{8}$ B. J. Geurts, "Inverse modeling for large-eddy simulation," Phys. Fluids 9, 3585-3588 (1997).

${ }^{9}$ S. Stolz and N. A. Adams, "An approximate deconvolution procedure for large-eddy simulation," Phys. Fluids 11, 16991701 (1999)

${ }^{10}$ J. G. M. Kuerten, "Subgrid modeling in particle-laden channel flow," Phys. Fluids 18, 025108 (2006).

${ }^{11}$ M. Germano, U. Piomelli, P. Moin, and W. H. Cabot, “A dynamic subgrid-scale eddy viscosity model," Phys. Fluids A 3, 1760-1765 (1991).

${ }^{12}$ B. J. Geurts and D. D. Holm, "Regularization modeling for large-eddy simulation," Phys. Fluids 15, L13 (2003).

${ }^{13}$ S. Stolz, N. A. Adams, and L. Kleiser, "An approximate deconvolution model for a large-eddy simulation with application to incompressible wall-bounded flows," Phys. Fluids 13, 997-1015 (2001).

${ }^{14}$ C. Marchioli, M. V. Salvetti, and A. Soldati, "Appraisal of energy recovering sub-grid scale models for large-eddy simulation of turbulent dispersed flows," Acta Mech. 201, 277-296 (2008).

${ }^{15}$ W. R. Michałek, R. Liew, J. G. M. Kuerten, and J. C. H. Zeegers, "LES of droplet-laden non-isothermal channel flow," J. Phys.: Conf. Ser. 318, 042056 (2011).

${ }^{16}$ G. Jin and G.-W. He, "A nonlinear model for the subgrid timescale experienced by heavy particles in large eddy simulation of isotropic turbulence with a stochastic differential equation,” New J. Phys. 15, 035011 (2013).

${ }^{17}$ C. Gobert, "Analytical assessment of models for large eddy simulation of particle laden flow," J. Turbul. 11, N23 (2010).

${ }^{18}$ B. Shotorban and F. Mashayek, "Modeling subgrid-scale effects on particles by approximate deconvolution," Phys. Fluids 17, 081701 (2005)

${ }^{19}$ B. Shotorban and F. Mashayek, "A stochastic model for particle motion in large-eddy simulation,” J. Turbul. 7, N18 (2006)

${ }^{20}$ B. Shotorban, K. K. Q. Zhang, and F. Mashayek, "Improvement of particle concentration prediction in large-eddy simulation by defiltering," Int. J. Heat Mass Transfer 50, 3728-3739 (2007).

${ }^{21}$ B. J. Geurts and J. G. M. Kuerten, "Ideal stochastic forcing for the motion of particles in large-eddy simulation extracted from direct numerical simulation of turbulent channel flow,” Phys. Fluids 24, 081702 (2012).

${ }^{22}$ J. Pozorski and S. V. Apte, "Filtered particle tracking in isotropic turbulence and stochastic modeling of subgrid-scale dispersion," Int. J. Multiphase Flow 35(2), 118-128 (2009).

${ }^{23}$ D. J. Thomson, "Criteria for the selection of stochastic models of particle trajectories in turbulent flows," J. Fluid Mech. 180, 529-556 (1987).

${ }^{24}$ L. Kleiser and U. Schumann, "Treatment of incompressibility and boundary conditions in 3-D numerical spectral simulations of plane channel flows," in Proceedings of the Third GAMM-Conference on Numerical Methods in Fluid Mechanics, edited by E. H. Hirschel (Vieweg, Braunschweig, 1980), pp. 165-173.

${ }^{25}$ B. V. Ramarao, C. Tien, and S. Mohan, "Calculation of single fiber efficiencies for interception and impaction with superposed Brownian motion," J. Aerosol Sci. 25, 295-313 (1994).

${ }^{26}$ U. Piomelli and E. Balaras, "Wall-layer models for large-eddy simulations," Annu. Rev. Fluid Mech. 34, 349-374 (2002).

${ }^{27}$ S. Hoyas and J. Jiménez, "Reynolds number effects on the Reynolds-stress budgets in turbulent channels," Phys. Fluids 20, 101511 (2008).

${ }^{28}$ F. Bianco, S. Chibbaro, C. Marchioli, M. V. Salvetti, and A. Soldati, "Intrinsic filtering errors of Lagrangian particle tracking in LES flow fields," Phys. Fluids 24, 045103 (2012). 